\title{
TROY is a promising prognostic biomarker in patients with colorectal cancer
}

\author{
MITSUAKI NISHIOKA ${ }^{1}$, YUTAKA SUEHIRO ${ }^{2}$, KOUHEI SAKAI ${ }^{2,3}$, TOSHIHIKO MATSUMOTO $^{2}$, \\ NAOKO OKAYAMA ${ }^{1}$, HIDEKAZU MIZUNO ${ }^{1}$, KOJI UENO ${ }^{4}$, NOBUAKI SUZUKI ${ }^{5}$, SHINICHI HASHIMOTO ${ }^{6}$, \\ TARO TAKAMI $^{6}$, SHOICHI HAZAMA ${ }^{7}$, HIROAKI NAGANO ${ }^{5}$, ISAO SAKAIDA ${ }^{6}$ and TAKAHIRO YAMASAKI ${ }^{1,2}$ \\ ${ }^{1}$ Division of Laboratory, Yamaguchi University Hospital; ${ }^{2}$ Department of Oncology and Laboratory Medicine, \\ Yamaguchi University Graduate School of Medicine, Ube, Yamaguchi 755-8505; ${ }^{3}$ Department of Gastroenterology, \\ Showa Hospital, Shimonoseki, Yamaguchi 750-0059; Departments of ${ }^{4}$ Surgery and Clinical Science, ${ }^{5}$ Gastroenterological, \\ Breast and Endocrine Surgery, ${ }^{6}$ Gastroenterology and Hepatology, and ${ }^{7}$ Translational Research and Developmental \\ Therapeutics Against Cancer, Yamaguchi University Graduate School of Medicine, Ube, Yamaguchi 755-8505, Japan
}

Received June 12, 2017; Accepted December 7, 2017

DOI: $10.3892 / \mathrm{ol} .2018 .8079$

\begin{abstract}
Tumor necrosis factor receptor superfamily member $19(T R O Y)$ is involved in the Wnt/ $\beta$-catenin signaling pathway and interacts with leucine-rich repeat containing G-proteincoupled receptor 5 (LGR5), which is a well-known biomarker of cancer stem cells and a prognostic marker of colorectal cancer (CRC). Because there have been no studies to evaluate the prognostic significance of TROY, we performed the present study to determine whether TROY can be a prognostic biomarker in CRC patients. We evaluated TROY expression levels in $100 \mathrm{CRC}$ tissues by quantitative real-time PCR and investigated the association of TROY expression levels with clinicopathologic features. Cancer stage and TROY expression level were found to be independent prognostic factors of disease-free survival. Moreover, TROY overexpression was the sole independent prognostic factor of disease-free survival in patients with stage II and III CRC. These results suggest that analysis of TROY might help predict clinical outcome in patients with CRC. To support our findings, confirmatory studies using independent data sets are needed.
\end{abstract}

Correspondence to: Dr Yutaka Suehiro, Department of Oncology and Laboratory Medicine, Yamaguchi University Graduate School of Medicine, 1-1-1 Minami-Kogushi, Ube, Yamaguchi 755-8505, Japan

E-mail: ysuehiro@yamaguchi-u.ac.jp

Abbreviations: CRC, colorectal cancer; DFS, disease-free survival; LGR5, leucine-rich-repeat-containing G-protein-coupled receptor 5; ROC, receiver operating characteristic; TROY, TNF receptor superfamily member 19 (TNFRSF19, TAJ)

Key words: colorectal cancer, leucine-rich repeat containing G-protein-coupled receptor 5, prognosis, tumor necrosis factor receptor superfamily member 19 , Wnt pathway

\section{Introduction}

Colorectal cancer (CRC) is the third most common cancer and the fourth leading cause of cancer-related deaths worldwide, accounting for roughly 1.36 million new people and 690,000 deaths per year in 2012 (1). The outcome of patients with CRC is related to the stage at diagnosis: The 5-year relative survival is $95.0 \%$ for CRC patients with stage I, $83.3 \%$ for those with stage II, $77.4 \%$ for those with stage III, and $16.9 \%$ for those with stage IV (2). Although adjuvant chemotherapy is recommended for CRC with high risk of relapse, its administration to patients with stage II and III $\mathrm{CRC}$, in which the 5 -year relapse rates are approximately 20 and $35-50 \%$, respectively, remains controversial $(3,4)$. Because patients with CRC that is identical in grade and stage often have significantly different clinical outcomes or responses to therapy and there are no established biomarkers to predict relapse, improved tumor classification is needed for CRC, especially for stage II and III CRC.

The Wnt/ $\beta$-catenin signaling pathway plays an essential role in the development of various tumors $(5,6)$. It is well known that the Wnt/ $\beta$-catenin signaling pathway is activated in most sporadic CRC (up to 80\%) (7). Recently, LGR5 (leucine-rich repeat containing G-protein-coupled receptor 5) was identified as the target gene of the Wnt/ $\beta$-catenin signaling pathway $(8,9)$. LGR5 is reported to be a biomarker of cancer stem cells of CRC and stem cells in adult intestinal crypts $(10,11)$. TROY (tumor necrosis factor receptor superfamily member 19 , TNFRSF19, TAJ) is a type I cell surface receptor protein containing the highly conserved TNFR cysteine-rich motifs in the extracellular domain and a TNF receptor-associated factor (TRAF)-binding sequence in the large cytoplasmic domain required for signaling (12). Recently, TROY was reported to be a $\beta$-catenin target gene and to form a complex with LGR5 in cellular membranes (13). Although overexpression of TROY is observed in CRC cell lines (14-17), its clinical significance for CRC is poorly understood. Because TROY might be a possible prognostic biomarker of CRC, we performed this study to 
investigate the clinical significance of TROY in patients with CRC and compared its clinical usefulness to that of LGR5.

\section{Materials and methods}

Materials. We collected 100 CRC tissues from 100 patients who underwent surgical treatment at the Department of Gastroenterological, Breast and Endocrine Surgery, Yamaguchi University Graduate School of Medicine between March 2000 and May 2008. From them, 36 matched normal-appearing mucosa tissues were also collected from a site distal to the resected materials. All samples were immediately frozen in liquid nitrogen after sample collection from surgically resected materials and then stored at $-80^{\circ} \mathrm{C}$. Total RNA was isolated using the AllPrep DNA/RNA Mini Kit (QIAGEN). The extracted total RNA was reverse transcribed into single-stranded cDNA using a High-Capacity cDNA Archive Kit (Applied Biosystems; Thermo Fisher Scientific, Inc., Waltham, MA, USA).

The clinicopathologic characteristics of the CRC patients are shown in Table I. The patient population consisted of 51 men and 49 women with a mean age of 66.9 years (range, 38-92 years). According to the staging system of the International Union Against Cancer (UICC) (18), 14 patients were stage I, 36 patients were stage II, 30 patients were stage III, and 20 patients were stage IV. After surgical treatments, 66 patients were treated with adjuvant chemotherapies using tegafur/uracil, cisplatin, or irinotecan, and 34 patients did not receive any adjuvant chemotherapy. This study was approved by the Institutional Review Board of Yamaguchi University Hospital (approval number: H28-073), and written informed consent was obtained from each patient before inclusion in the study.

Quantitative real-time PCR. Quantitative real-time PCR was performed using TaqMan Gene Expression Master Mix (Applied Biosystems; Thermo Fisher Scientific, Inc.). The Applied Biosystems catalog numbers of the primer and probe set for the endogenous control ( $\beta$-actin) and target genes were 4326315E for $\beta$-actin, Hs00969422_m1 for LGR5, and Hs00218634_m1 for TROY. Quantitative real-time PCR was performed on an ABI Prism 7900HT Sequence Detection System (Applied Biosystems; Thermo Fisher Scientific, Inc.). PCR cycling conditions included preheating at $50^{\circ} \mathrm{C}$ for $2 \mathrm{~min}$ for optimal UDG (uracil-DNA glycosylase) enzyme activity and at $95^{\circ} \mathrm{C}$ for $10 \mathrm{~min}$ for AmpliTaq Gold DNA polymerase and UP enzyme activation followed by 40 cycles of denaturation at $95^{\circ} \mathrm{C}$ for $15 \mathrm{sec}$ and annealing at $60^{\circ} \mathrm{C}$ for $1 \mathrm{~min}$. All reactions were carried out in a $20-\mu 1$ reaction volume in triplicate. The mRNA expression level was determined using the $2^{-\triangle \Delta C T}$ method, in which relative quantification of mRNA expression level was calculated using $\beta$-actin as the internal reference (19).

Statistical analysis. Data analyses were performed using StatFlex v6 (Artech Co., Ltd., Osaka, Japan). Student's t-test, Fisher's exact test, one-way analysis of variance (ANOVA) followed by Tukey multiple comparison test, two-tailed Spearman's test, Kaplan-Meier analysis, stepwise multiple regression analysis, or Cox proportional hazard regressions analysis was used. Disease-free survival (DFS) time was defined as the length of time after the primary surgical treatment for CRC during which

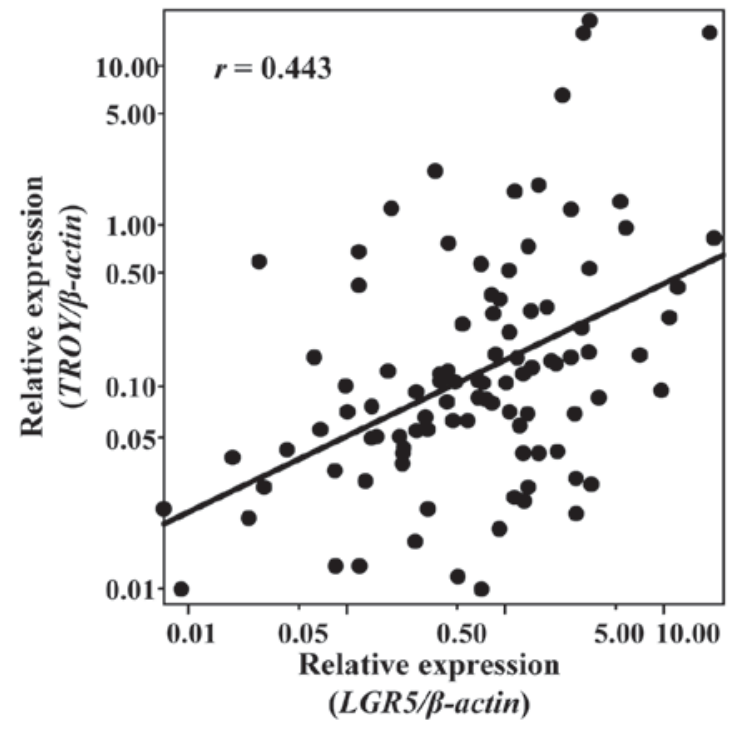

Figure 1. Correlation between TROY and LGR5 expression levels. Each sample is indicated by a black circle. TROY, tumor necrosis factor receptor superfamily member 19; LGR5, leucine-rich repeat containing G-proteincoupled receptor 5 .

no recurrence was detected. The follow-up period ended on June 30, 2016. Before the analysis, a logarithmic transformation was performed on $L G R 5$ and TROY mRNA expression values to obtain normally distributed data sets. $\mathrm{P}<0.05$ was considered to indicate a statistically significant difference.

\section{Results}

LGR5 and TROY mRNA expression levels in the CRC and non-tumor specimens. There was a positive correlation of expression level between TROY and $L G R 5$ ( $r=0.443, \mathrm{P}<0.001$ by two-tailed Spearman's test) (Fig. 1). LGR5 mRNA expression levels were significantly higher in the CRC tissues of each stage except for stage I than those in the normal-appearing mucosa tissues $(\mathrm{P}<0.001$ by one-way ANOVA; both $\mathrm{P}<0.01$ by Tukey multiple comparison test) (Fig. 2A). TROY mRNA expression levels were significantly higher in the CRC tissues of each stage than those in the normal-appearing mucosa tissues $(\mathrm{P}<0.001$ by one-way ANOVA; both $\mathrm{P}<0.01$ by Tukey multiple comparison test) (Fig. 3A).

Prognostic significance of LGR5. LGR5 expression levels were slightly higher in the relapse group than in the diseasefree group ( $\mathrm{P}=0.058$ by Student's t-test) (Fig. 2B). Receiver operator characteristic (ROC) curves and sensitivity and specificity curves were plotted using LGR5 mRNA expression values for differentiating between the disease-free condition and relapse. As the crossover of the sensitivity and specificity curves was 0.739 , we divided the CRC patients into 2 groups on this basis: the $L G R 5$-high group $(\geq 0.739)$ and the LGR5-low (<0.739) group. Univariate analysis showed CRC patients in the LGR5-low group tended to have improved DFS as compared with those in the $L G R 5$-high group ( $\mathrm{P}=0.055$ by log-rank test) (Fig. 2C). In contrast, there was no association between the $L G R 5$ expression level and overall survival status ( $\mathrm{P}=0.37$ by Student's t-test) (data not shown). 
Table I. Relation between clinicopathologic factors and patient outcomes.

\begin{tabular}{|c|c|c|c|c|c|c|c|}
\hline \multirow[b]{2}{*}{ Factor } & \multirow[b]{2}{*}{$\mathrm{n}$} & \multicolumn{3}{|c|}{ Relapse status } & \multicolumn{3}{|c|}{ Survival status } \\
\hline & & Disease-free (n) & Relapse (n) & P-value & Survival (n) & Non-survival (n) & P-value \\
\hline \multicolumn{8}{|l|}{ Sex } \\
\hline Male & 51 & 36 & 15 & 0.9000 & 38 & 13 & 0.5500 \\
\hline Female & 49 & 34 & 15 & & 39 & 10 & \\
\hline $\begin{array}{l}\text { Age in years } \\
(\text { mean } \pm \mathrm{SD} / \text { range })\end{array}$ & $\begin{array}{c}66.9 \pm 11.4 / \\
38-92\end{array}$ & $\begin{array}{c}67.3 \pm 11.6 / \\
38-92\end{array}$ & $\begin{array}{c}66.1 \pm 11.2 / \\
48-89\end{array}$ & 0.4900 & $\begin{array}{c}67.3 \pm 11.4 / \\
38-92\end{array}$ & $\begin{array}{c}65.5 \pm 11.7 / \\
49-89\end{array}$ & 0.5100 \\
\hline \multicolumn{8}{|l|}{ pStage } \\
\hline I & 14 & 14 & 0 & NA & 14 & 0 & NA \\
\hline II & 36 & 27 & 9 & 0.0390 & 30 & 6 & 0.1700 \\
\hline III & 30 & 21 & 9 & 0.0220 & 24 & 6 & 0.0720 \\
\hline IV & 20 & 8 & 12 & 0.0003 & 9 & 11 & 0.0007 \\
\hline \multicolumn{8}{|l|}{$\mathrm{pT}$} \\
\hline 1 & 8 & 8 & 0 & NA & 8 & 0 & NA \\
\hline 2 & 9 & 8 & 1 & 0.3300 & 8 & 1 & 0.3300 \\
\hline 3 & 70 & 48 & 22 & 0.0610 & 54 & 16 & 0.1300 \\
\hline 4 & 13 & 6 & 7 & 0.0110 & 7 & 6 & 0.0230 \\
\hline \multicolumn{8}{|l|}{$\mathrm{pN}$} \\
\hline 0 & 55 & 43 & 12 & NA & 47 & 8 & NA \\
\hline 1 & 23 & 17 & 6 & 0.6800 & 17 & 6 & 0.2300 \\
\hline 2 & 22 & 10 & 12 & 0.0051 & 13 & 9 & 0.0120 \\
\hline \multicolumn{8}{|l|}{$\mathrm{pM}$} \\
\hline 0 & 80 & 62 & 18 & NA & 68 & 12 & NA \\
\hline 1 & 20 & 8 & 12 & 0.0011 & 9 & 11 & 0.0001 \\
\hline \multicolumn{8}{|l|}{$\begin{array}{l}\text { Lymphatic vessel } \\
\text { invasion }\end{array}$} \\
\hline Negative & 12 & 12 & 0 & NA & 12 & 0 & NA \\
\hline Positive & 88 & 58 & 30 & 0.0160 & 65 & 23 & 0.0440 \\
\hline \multicolumn{8}{|l|}{ Vascular invasion } \\
\hline Negative & 31 & 26 & 5 & NA & 27 & 4 & NA \\
\hline Positive & 69 & 44 & 25 & 0.0430 & 50 & 19 & 0.1100 \\
\hline \multicolumn{8}{|l|}{ Liver metastasis } \\
\hline Negative & 86 & 63 & 23 & NA & 70 & 16 & NA \\
\hline Positive & 14 & 7 & 7 & 0.0780 & 7 & 7 & 0.0096 \\
\hline \multicolumn{8}{|l|}{ Peritoneal metastasis } \\
\hline Negative & 91 & 66 & 25 & NA & 74 & 17 & NA \\
\hline Positive & 9 & 4 & 5 & 0.0800 & 3 & 6 & 0.0011 \\
\hline \multicolumn{8}{|l|}{ Histopathological type } \\
\hline $\begin{array}{l}\text { Well differentiated } \\
\text { adenocarcinoma }\end{array}$ & 29 & 25 & 4 & NA & 25 & 4 & NA \\
\hline Others & 71 & 44 & 26 & 0.0210 & 51 & 19 & 0.1500 \\
\hline \multicolumn{8}{|c|}{$\begin{array}{l}\text { Postoperative adjuvant } \\
\text { chemotherapy }\end{array}$} \\
\hline Not performed & 34 & 31 & 3 & NA & 30 & 4 & NA \\
\hline Performed & 66 & 39 & 27 & 0.0009 & 47 & 19 & 0.0550 \\
\hline
\end{tabular}

pStage, pathological stage; pT, pathological tumor classification; pN, lymph node metastasis; pM, distant metastasis; NA, not applicable; SD, standard deviation. 
A

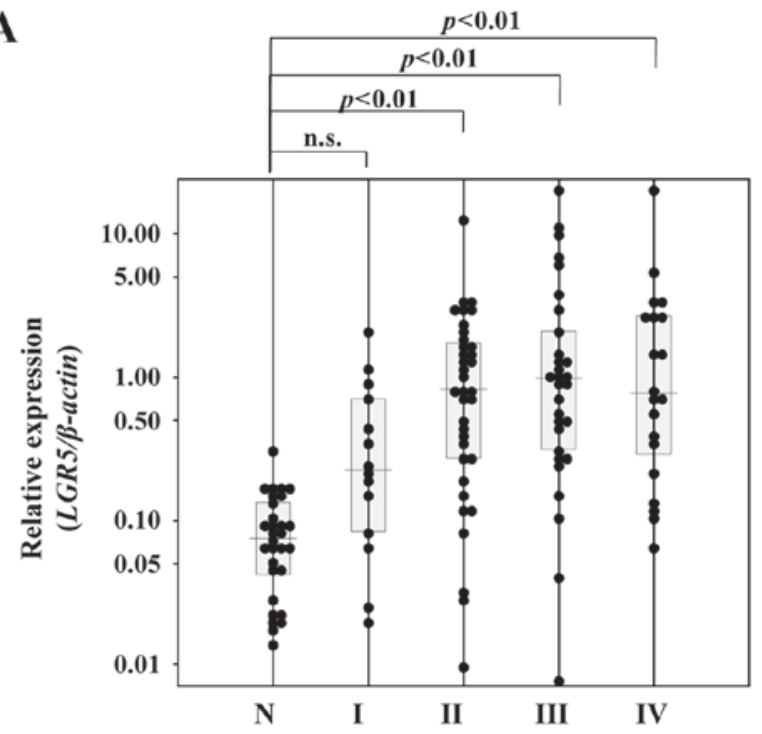

B

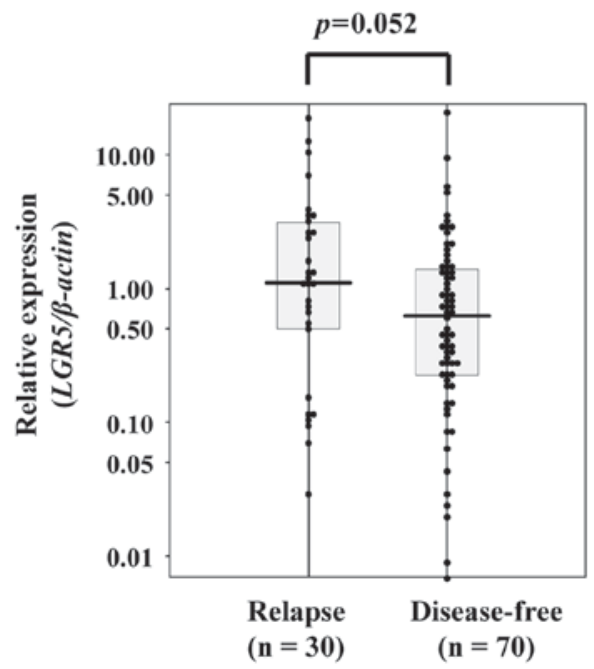

C

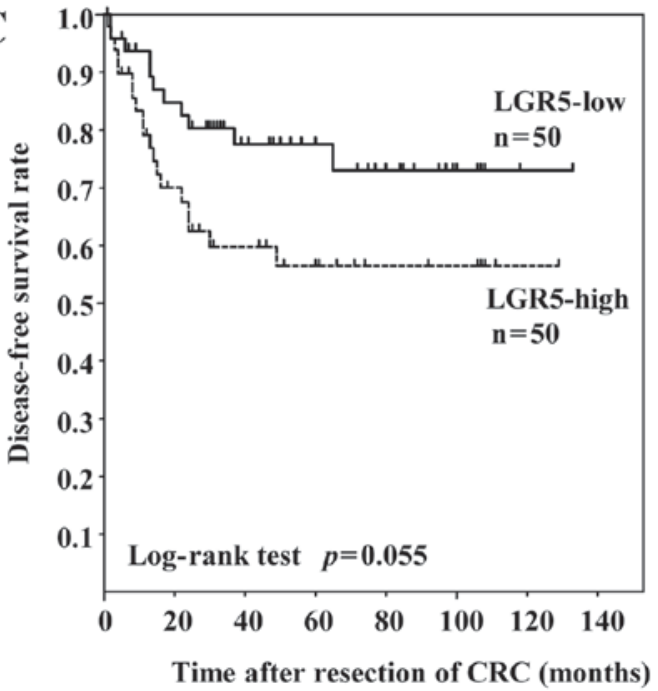

D

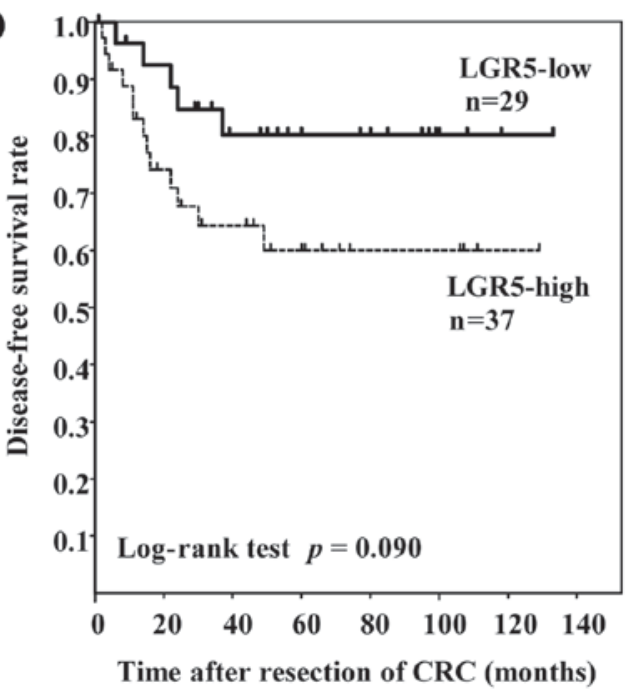

Figure 2. (A) Distribution of LGR5 mRNA expression levels in normal-appearing mucosa specimens and CRC specimens of each stage. (B) LGR5 levels in the relapse group and the disease-free group. (C) Disease-free survival in all CRC patients according to LGR5 expression status. (D) The horizontal lines represent the median level in each group. N, non-tumor specimens; n.s., not significant; I, II, III, and IV, pathological stages of CRC. CRC, colorectal cancer; LGR5, leucine-rich repeat containing G-protein-coupled receptor 5.

TROY as a prognostic biomarker of CRC. There was a significant difference in the expression level of TROY between the disease-free and recurrence groups $(\mathrm{P}=0.0004$ by Student's t-test) (Fig. 3B). ROC analysis revealed the area under the ROC curve to be 0.694 , and an optimal cut-off point to discriminate between the disease-free and recurrence groups was 0.15 according to the crossover of the sensitivity and specificity curves, resulting in a sensitivity of $60.0 \%$ and a specificity of $77.1 \%$. Univariate survival analysis showed patients with high TROY expression $(\mathrm{n}=36)$ had a significantly shorter duration of DFS than those with low TROY expression $(n=64)(P=0.0003$ by log-rank test) (Fig. 3C), and this association was also observed when patients were limited to stage II and III CRC ( $\mathrm{P}=0.0026$ by log-rank test) (Fig. 3D). Multivariate analysis identified stage and TROY mRNA level as the independent predictive factors of DFS (Table II). In contrast, there was no association between TROY mRNA
Table II. Results of Cox proportional hazards regression analysis of risk factors for recurrence in patients with all stages colorectal cancer $(n=100)$.

\begin{tabular}{lccc}
\hline Variable & P-value & HR & $95 \%$ CI \\
\hline Sex $^{\text {a }}$ & 0.6378 & 0.837 & $0.399-1.757$ \\
Age & 0.5711 & 1.010 & $0.976-1.046$ \\
pStage & 0.0004 & 2.288 & $1.444-3.624$ \\
LGR5 mRNA level & 0.6209 & 0.856 & $0.463-1.583$ \\
TROY mRNA level & 0.0039 & 2.364 & $1.317-4.241$
\end{tabular}

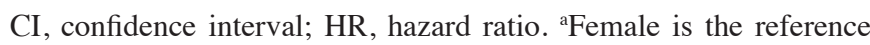
category. LGR5, leucine-rich repeat containing G-protein-coupled receptor 5; TROY, tumor necrosis factor receptor superfamily member 19 . 
A

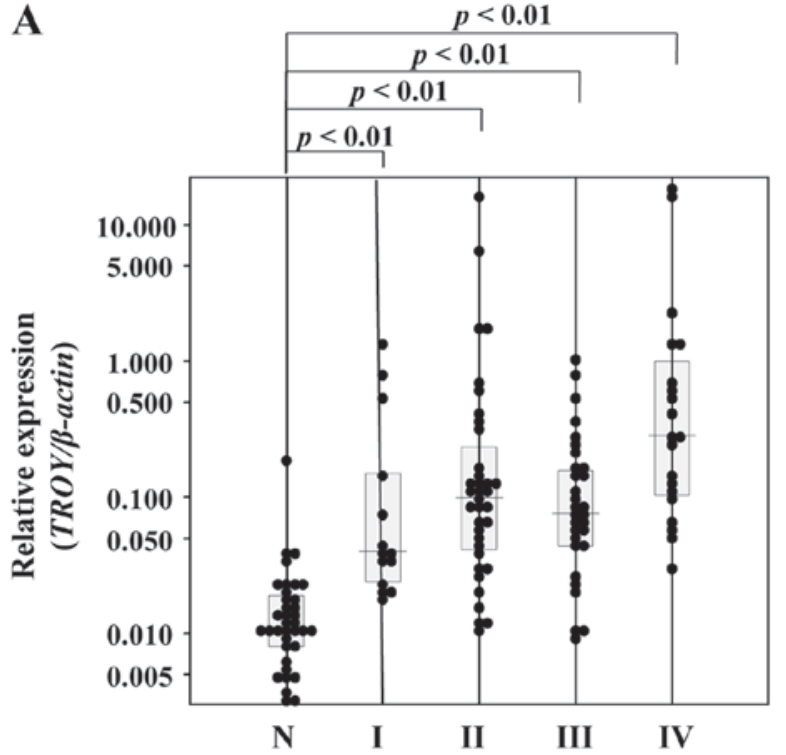

B

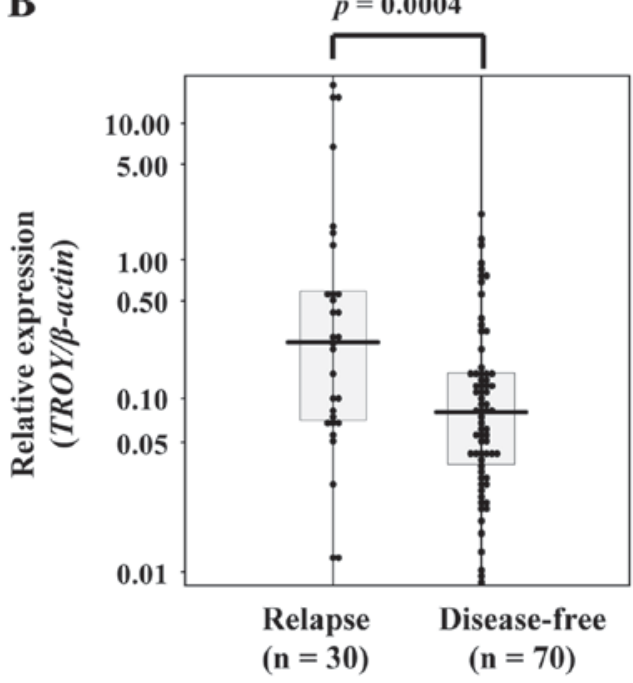

C

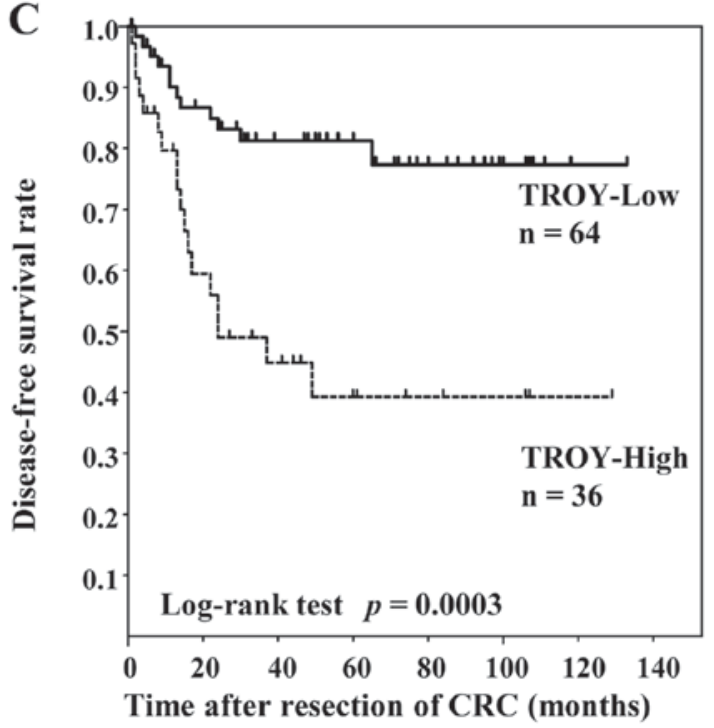

D

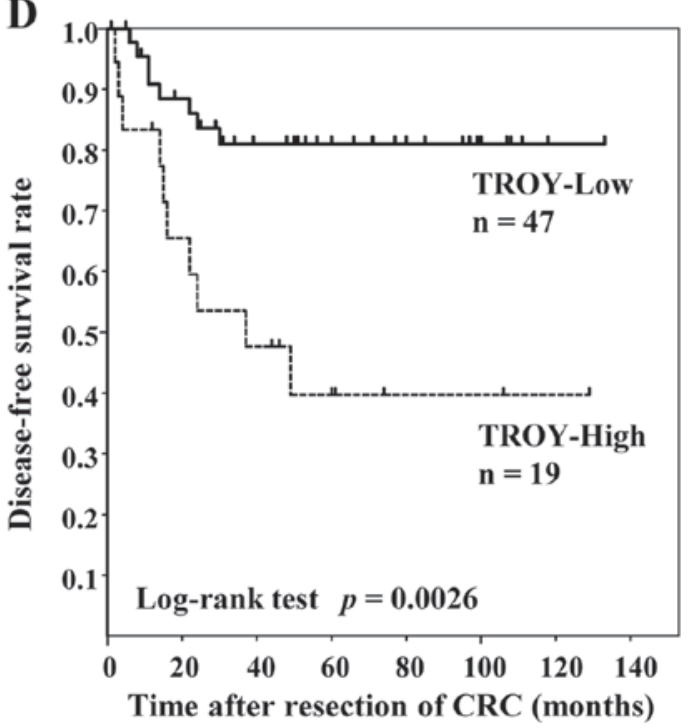

Figure 3. (A) Distribution of TROY mRNA expression levels in normal-appearing mucosa specimens and CRC specimens of each stage. (B) TROY levels in the relapse group and the disease-free group. (C) Kaplan-Meier plots of disease-free survival in patients with all stages of CRC (D) and in patients with stage II and III CRC according to TROY expression levels. TROY, tumor necrosis factor receptor superfamily member 19; CRC, colorectal cancer.

level and overall survival status ( $\mathrm{P}=0.14$ by Student's $t$-test $)$ (data not shown).

\section{Discussion}

In the present study, LGR 5 mRNA expression levels were significantly higher in the CRC tissues of stages II-IV than those in the normal-appearing mucosa tissues, and CRC patients with higher $L G R 5$ expression tended to have unfavorable outcomes compared with those with lower LGR5 expression. $L G R 5$ overexpression has been reported in several cancers including hepatocellular carcinoma, ovarian cancer, cervical cancer, and basal cell carcinoma (20-23). Regarding CRC, many study groups reported that LGR5 is overexpressed in CRC tissues and is associated with unfavorable outcome in CRC patients (24-27). In the present study, although univariate analysis showed overexpression of $L G R 5$ to be a possible biomarker of unfavorable outcome of $\mathrm{CRC}$, its clinical usefulness in predicting relapse of CRC was not as expected. Thus, we continued searching for other biomarkers including TROY.

In the present study, the overexpression of TROY was found to be an independent prognostic marker of DFS in all stages of CRC and even when limited only to stage II and III CRC. To date, this is, to our knowledge, the first report in the world of TROY as an independent prognostic marker of DFS of CRC. TROY has unique aspects in the Wnt signaling pathway. TROY has the potential of being a negative feedback mechanism in the canonical Wnt pathway. Because TROY is one of the $\beta$-catenin/TCF4 target genes, TROY expression is increased in a Wnt-signalingdependent manner; however, TROY also reduces Wnt signaling by destabilizing LRP6 (28). Thus, we speculate that the overexpression of TROY observed in the present study may reflect a failure of the negative feedback of TROY in Wnt signaling or a protective effect of TROY to suppress Wnt signaling. The 
function of TROY in CRC is poorly understood. One research group reported that TROY is significantly up-regulated in neoplastic tissues from mice during intestinal tumorigenesis and is produced specifically by fast-cycling intestinal stem cells (13). Another research group suggested that TROY can contribute to the initiation or progression of colorectal tumors with deregulated $\beta$-catenin activity (17). Regarding the prognostic significance of TROY, one research group reported no increase in TROY mRNA in CRC specimens in a small sample of patients $(n=20)(13)$. In contrast, another group reported an increase in TROY mRNA expression in 4 of 8 CRC tissues but did not investigate its prognostic significance (17). Because the number of samples analyzed in both reports was small, they were insufficient to evaluate the prognostic significance of the expression level of TROY. Further studies are required to confirm the association of the higher expression of TROY with outcome in patients with CRC. In cancers other than CRC, higher expression of TROY correlates with increasing glial tumor grade (15), glioblastoma cell invasion, and unfavorable outcome of glioblastoma $(16,29)$. These reports may support our finding of TROY as a possible prognostic marker in CRC. However, we realize that limitations exist in our study: We did not measure protein levels of TROY and did not investigate its localization because of an insufficient amount of tissue samples. Thus, further studies incorporating investigation of these issues are required to prove TROY as a true prognostic factor in CRC.

In conclusion, TROY may be a promising and better biomarker of predictive relapse of CRC than LGR5. To support our findings, confirmatory studies using independent data sets are needed. In addition, to improve the outcome of patients with CRC, further studies are required to investigate whether the evaluation of TROY expression level can be useful in determining whether to introduce adjuvant chemotherapy especially in patients with stage II and III CRC.

\section{Acknowledgements}

The authors would like to thank Reo Kawano (Center for Clinical Research, Yamaguchi University Hospital) for his help with the statistical analysis. The present work was supported by JSPS KAKENHI Grant Numbers JP25931034, JP26893169 and JP16K19195 (to M.N.).

\section{References}

1. GLOBOCAN 2012. Cited 11 June 2017. Available from URL: http://globocan.iarc.fr/.

2. CANCERSTATISTICSINJAPAN '15. Cited 11 June 2017. Available from URL: http://ganjoho.jp/en/professional/ statistics/brochure/2015_en.html

3. Böckelman C, Engelmann BE, Kaprio T, Hansen TF and Glimelius B: Risk of recurrence in patients with colon cancer stage II and III: A systematic review and meta-analysis of recent literature. Acta Oncol 54: 5-16, 2015.

4. Verhoeff SR, van Erning FN, Lemmens VE, de Wilt JH and Pruijt JF: Adjuvant chemotherapy is not associated with improved survival for all high-risk factors in stage II colon cancer. Int J Cancer 139: 187-193, 2016.

5. Reya $\mathrm{T}$ and Clevers $\mathrm{H}$ : Wnt signalling in stem cells and cancer. Nature 434: 843-850, 2005

6. MacDonald BT, Tamai K and He X: Wnt/beta-catenin signaling: Components, mechanisms, and diseases. Dev Cell 17: 9-26, 2009

7. Schneikert J and Behrens J: The canonical Wnt signalling pathway and its APC partner in colon cancer development. Gut 56: 417-425, 2007.
8. Barker N, van Es JH, Kuipers J, Kujala P, van den Born M, Cozijnsen M, Haegebarth A, Korving J, Begthel H, Peters PJ and Clevers H: Identification of stem cells in small intestine and colon by marker gene Lgr5. Nature 449: 1003-1007, 2007.

9. Barker $\mathrm{N}$ and Clevers $\mathrm{H}$ : Leucine-rich repeat-containing G-protein-coupled receptors as markers of adult stem cells. Gastroenterology 138: 1681-1696, 2010.

10. Hirsch D, Barker N, McNeil N, Hu Y, Camps J, McKinnon K, Clevers H, Ried T and Gaiser T: LGR5 positivity defines stemlike cells in colorectal cancer. Carcinogenesis 35: 849-858, 2014.

11. Barker N, Ridgway RA, van Es JH, van de Wetering M, Begthel H, van den Born M, Danenberg E, Clarke AR, Sansom OJ and Clevers H: Crypt stem cells as the cells-of-origin of intestinal cancer. Nature 457: 608-611, 2009.

12. Hu S, Tamada K, Ni J, Vincenz C and Chen L: Characterization of TNFRSF19, a novel member of the tumor necrosis factor receptor superfamily. Genomics 62: 103-107, 1999.

13. Fafilek B, Krausova M, Vojtechova M, Pospichalova V, Tumova L, Sloncova E, Huranova M, Stancikova J, Hlavata A, Svec J, et al: Troy, a tumor necrosis factor receptor family member, interacts with $\operatorname{lgr} 5$ to inhibit wnt signaling in intestinal stem cells. Gastroenterology 144: 381-391, 2013.

14. Spanjaard RA, Whren KM, Graves C and Bhawan J: Tumor necrosis factor receptor superfamily member TROY is a novel melanoma biomarker and potential therapeutic target. Int J Cancer 120: 1304-1310, 2007.

15. Paulino VM, Yang Z, Kloss J, Ennis MJ, Armstrong BA, Loftus JC and Tran NL: TROY (TNFRSF19) is overexpressed in advanced glial tumors and promotes glioblastoma cell invasion via Pyk2-Rac1 signaling. Mol Cancer Res 8: 1558-1567, 2010.

16. Loftus JC, Dhruv H, Tuncali S, Kloss J, Yang Z, Schumacher CA, Cao B, Williams BO, Eschbacher JM and Ross JT: TROY (TNFRSF19) promotes glioblastoma survival signaling and therapeutic resistance. Mol Cancer Res 11: 865-874, 2013.

17. Schön S, Flierman I, Ofner A, Stahringer A, Holdt LM, Kolligs FT and Herbst A: $\beta$-catenin regulates NF- $\kappa$ B activity via TNFRSF19 in colorectal cancer cells. Int J Cancer 135: 1800-1811, 2014.

18. Sobin LH, Gospodarowicz MK and Wittekind C, (eds): TNM classification of malignant tumours, 7th Edition. Hoboken WileyBlackwell: pp336, 2009.

19. Livak KJ and Schmittgen TD: Analysis of relative gene expression data using real-time quantitative PCR and the 2(-Delta Delta C(T)) method. Methods 25: 402-408, 2001.

20. Yamamoto Y, Sakamoto M, Fujii G, Tsuiji H, Kenetaka K, Asaka M and Hirohashi S: Overexpression of orphan G-proteincoupled receptor, Gpr49, in human hepatocellular carcinomas with beta-catenin mutations. Hepatology 37: 528-533, 2003.

21. Tanese K, Fukuma M, Yamada T, Mori T, Yoshikawa T, Watanabe W,Ishiko A, Amagai M,Nishikawa T and Sakamoto M: G-protein-coupled receptor GPR49 is up-regulated in basal cell carcinoma and promotes cell proliferation and tumor formation. Am J Pathol 173: 835-843, 2008.

22. Sun B, Ye X, Li Y and Zhang W: Lgr5 is a potential prognostic marker in patients with cervical carcinoma. Int J Clin Exp Pathol 8: 1783-1789, 2015.

23. Sun Y, Jia X and Wu X: High expressions of Lgr5 and ALDH1 in primary epithelial ovarian cancer correlate with advanced tumor stage and grade as well as poor prognosis of the patients. Gynecol Obstet Invest, 20 Jun 2015 (Epub ahead of print).

24. Takahashi H, Ishii H, Nishida N, Takemasa I, Mizushima T, Ikeda M, Yokobori T, Mimori K, Yamamoto H, Sekimoto M, et al: Significance of Lgr5(+ve) cancer stem cells in the colon and rectum. Ann Surg Oncol 18: 1166-1174, 2011.

25. Wu XS, Xi HQ and Chen L: Lgr5 is a potential marker of colorectal carcinoma stem cells that correlates with patient survival. World J Surg Oncol 10: 244, 2012.

26. Saigusa S, Inoue Y, Tanaka K, Toiyama Y, Kawamura M, Okugawa Y, Okigami M, Hiro J, Uchida K, Mohri Y and Kusunoki M: Significant correlation between LKB1 and LGR5 gene expression and the association with poor recurrence-free survival in rectal cancer after preoperative chemoradiotherapy. J Cancer Res Clin Oncol 139: 131-138, 2013.

27. Hsu HC, Liu YS, Tseng KC, Hsu CL, Liang Y, Yang TS, Chen JS, Tang RP, Chen SJ and Chen HC: Overexpression of Lgr5 correlates with resistance to 5-FU-based chemotherapy in colorectal cancer. Int J Colorectal Dis 28: 1535-1546, 2013.

28. Barker N, Tan S and Clevers H: Lgr proteins in epithelial stem cell biology. Development 140: 2484-2494, 2013.

29. Dhruv HD, Roos A, Tomboc PJ, Tuncali S, Chavez A, Mathews I, Berens ME, Loftus JC and Tran NL: Propentofylline inhibits glioblastoma cell invasion and survival by targeting the TROY signaling pathway. J Neurooncol 126: 397-404, 2016. 